\title{
Aktorisches Miniaturspektrometer für die Gassensorik
}

\author{
Martin Schädel ${ }^{1}$, Julia Baldauf ${ }^{1}$, Nicole Thronicke ${ }^{1}$, Dennis Mitrenga ${ }^{1}$, Dominik Karolewski ${ }^{1}$, Hans- \\ Georg Ortlepp ${ }^{1}$, Adrian Grewe ${ }^{2}$, Stefan Sinzinger ${ }^{2}$, Thomas Ortlepp ${ }^{1}$ \\ ${ }^{1}$ CiS Forschungsinstitut für Mikrosensorik GmbH, Konrad-Zuse-Straße 14, 99099 Erfurt, \\ Deutschland \\ ${ }^{2}$ Technische Universität IImenau, Institut für Mikro- und Nanotechnologien MacroNano® \\ Fachgebiet Technische Optik, Am Helmholtzring 1, 98693 IImenau, Deutschland
}

\section{Zusammenfassung}

Der Beitrag zeigt den bisherigen Zwischenstand der Entwicklung eines neuartigen MEMS-basierten Infrarot-Spektrometers für die Gassensorik. Dieses Spektrometer besteht aus diffraktiven optischen Elementen (DOE) mit einer gezielt hohen chromatischen Aberration, welche auf einer Aktormembran angeordnet sind, einem Blendensystem und Thermopilesensoren. Die DOE in Kombination mit den Blenden ermöglichen eine gezielte Auswahl des Spektralbereiches.

DOE-Design und Fertigung, sowie Membrandesign und Fertigung werden in diesem Beitrag detailliert erläutert.

Keywords: Gassensorik, optische Messverfahren

\section{Ziel der Entwicklung}

Das Ziel ist die Entwicklung eines Sensors für die Konzentrationsanalyse von Gasen und Gasgemischen auf dem Prinzip der Infrarotabsorption. Die im Fokus stehende Detektoreinheit nutzt eine innovative Methode für die Gewinnung eines kontinuierlichen Absorptionsspektrums. Diese Methode basiert auf der Kombination von diffraktiven optischen Elementen (DOE) mit gezielt hoher chromatischer Aberration, welche sich auf einem Aktor befinden, durch den der Abstand zwischen den DOE und dahinter befindlichen Blenden eingestellt werden kann. Je nach Abstand zwischen Blende und DOE passiert ein anderer Wellenlängenbereich die Blende und wird vom Thermopile erfasst. Durch die Kombination von Spule und Permanentmagnet kann der Abstand zwischen DOE und Blende mit Hilfe einer Aktuatormembran stufenlos eingestellt werden. Auf diese Weise kann ein kontinuierliches Absorptionsspektrum aufgenommen werden. Die Erweiterung auf mehrere Kanäle erlaubt das Erfassen eines mehrere Mikrometer breiten Spektralbereichs. Abb. 1 zeigt einen schematischen Schnitt des geplanten Gasmesssystems und der geplanten Infrarotdetektoreinheit mit durchstimmbarer spektraler Empfindlichkeit. Die infrarote Strahlung, durchläuft ein Gasgemisch und wird durch die beiden verschiedenen DOEs auf die darunter angebrachten Blenden fokussiert.
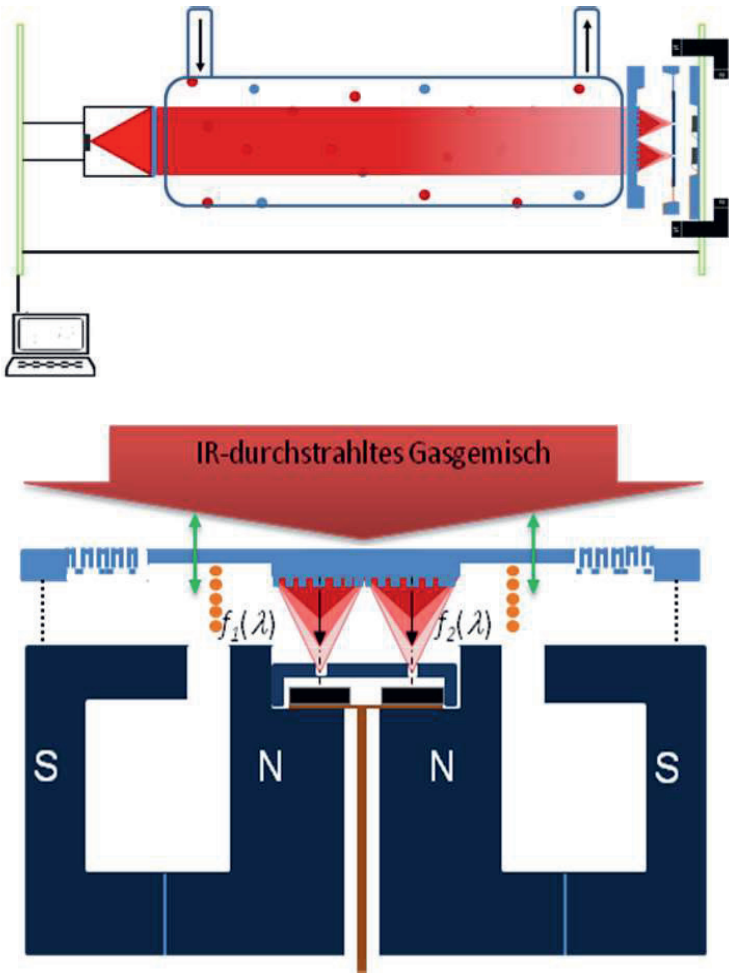

Abb. 1: Oben: Schematischer Schnitt der geplanten IR-Detektoreinheit mit Infrarotquelle, Gasmesszelle und Detektoreinheit, Unten: Detektoreinheit mit durchstimmbarer spektraler Empfindlichkeit. 


\section{Design und Fertigung der DOEs}

DOEs mit einer hohen chromatischen Aberration werden häufig für Messsysteme, welche nach dem chromatisch-konfokalen Prinzip zur Abstandsmessung arbeiten, eingesetzt [1]. Die für das chromatischkonfokale Messprinzip zu beachtenden Design-Kriterien (Arbeitsabstand, Messbereich, axiale und laterale Auflösung, erlaubte Verkippung des Testobjektes) [1] unterscheiden sich von den hier betrachteten Design-Kriterien für die DOEs und das Gesamtsystem.

Ausgehend von den Design-Parametern für die DOEs (Linsendurchmesser $\geq 1 \mathrm{~mm}$, Brennweite etwa $1 \mathrm{~mm}$, zentrale Wellenlänge 4,3 $\mu \mathrm{m}$, Verstimmbereich $\pm 250 \mu \mathrm{m}$, Substratdicke 300 $\mu \mathrm{m})$ wurden optische Simulationen auf Basis der entsprechenden DOE und Blenden mit Hilfe kommerzieller Software (Zemax) durchgeführt. Dabei wurde der Blendendurchmesser variiert, um den Einfluss verschiedener Systemparameter auf die Gesamtintensität und die Auflösung des Systems zu untersuchen.

Es wurden drei DOE (siehe Tab. 1) mit Linsendurchmessern von 1,5 mm simuliert.

Tab. 1: Parameter der simulierten DOEs

\begin{tabular}{|l|l|l|l|}
\hline & DOE1 & DOE2 & DOE3 \\
\hline Brennweite $(\lambda=4,3$ & 0,8 & 1,0 & 1,6 \\
$\mu \mathrm{m})$ & $\mathrm{mm}$ & $\mathrm{mm}$ & $\mathrm{mm}$ \\
\hline Wellenlängenbereich & 3,59 & 3,64 & 3,79 \\
& $\mu \mathrm{m}-$ & $\mu \mathrm{m}-$ & $\mu \mathrm{m}-$ \\
& 5,16 & 5,14 & 4,95 \\
& $\mu \mathrm{m}$ & $\mu \mathrm{m}$ & $\mu \mathrm{m}$ \\
\hline Numerische Apertur & 0,68 & 0,60 & 0,42 \\
\hline
\end{tabular}
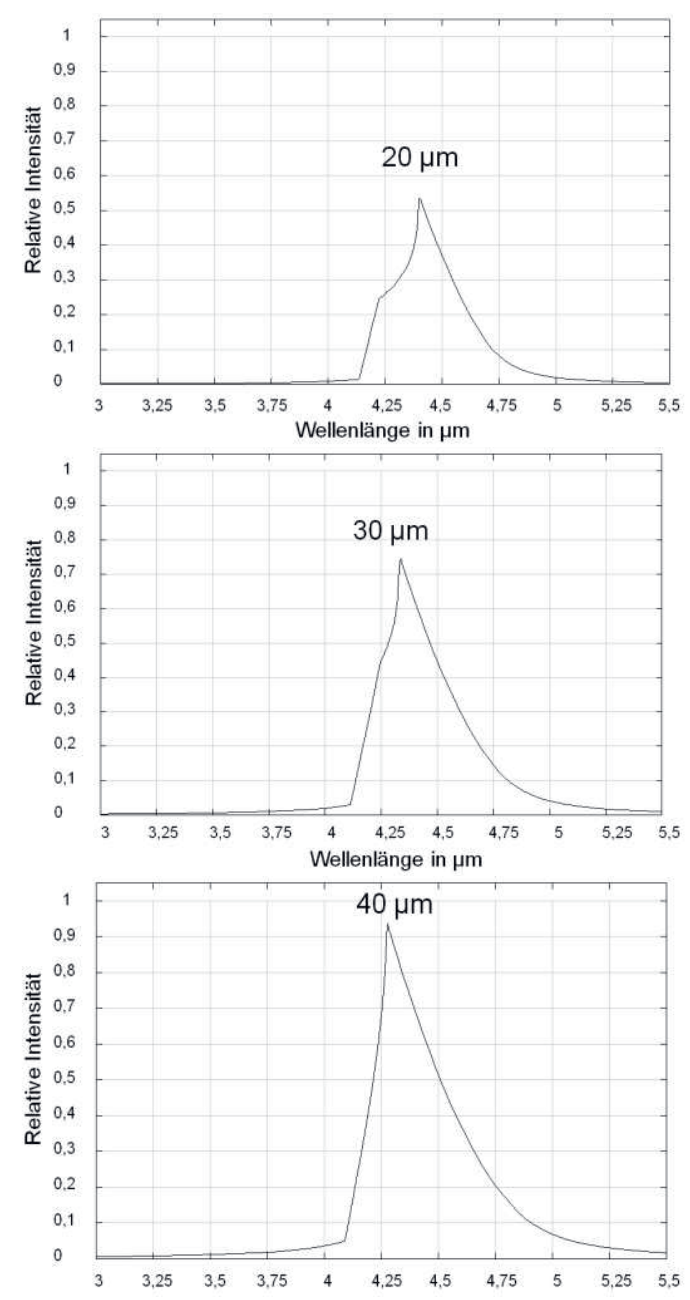

Abb. 2: Simulierte relative Intensität für DOE1 in Kombination mit verschiedenen Blenden.

Anhand der Simulationsergebnisse für verschiedene Blendendurchmesser wurden geeignete Blendendurchmesser evaluiert.

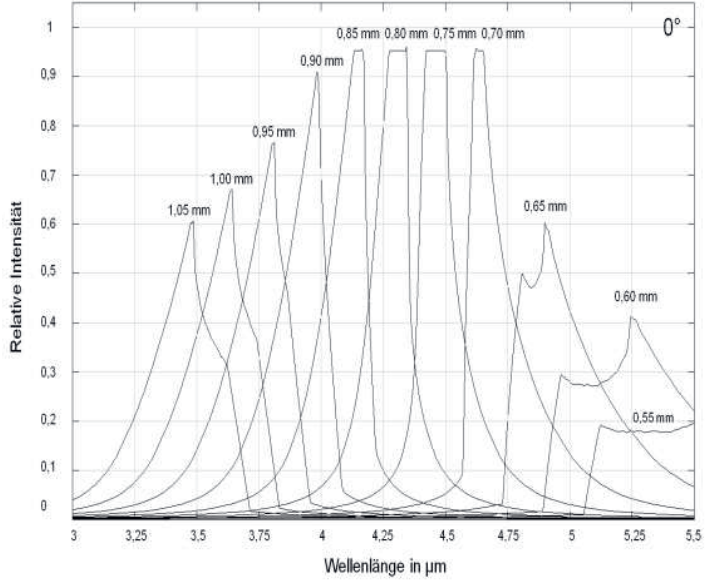

Abb. 3: Simulierte relative Intensität in Abhängigkeit von der Wellenlänge für ein System aus DOE1 und einer Blende von $30 \mu \mathrm{m}$ Durchmesser für verschiedene Abstände mit Lichteinfall unter einem objektseitigen Winkel von $0^{\circ}$. 
In Abb. 3 ist beispielhaft die simulierte relative Intensität in Abhängigkeit von der Wellenlänge für DOE1 und einer Blende von $30 \mu \mathrm{m}$ Durchmesser für verschiedene Abstände zwischen DOE und Blende aufgetragen. Anhand dieser Simulation ist zu erkennen, dass es theoretisch möglich ist die angestrebte Sensorfunktionalität auf Basis der Systemaufbaues zu realisieren.

Es wurde außerdem simuliert wie sich eine Änderung des objektseitigen Winkelbereiches auf die relative Intensität in Abhängigkeit von der Wellenlänge auswirkt.
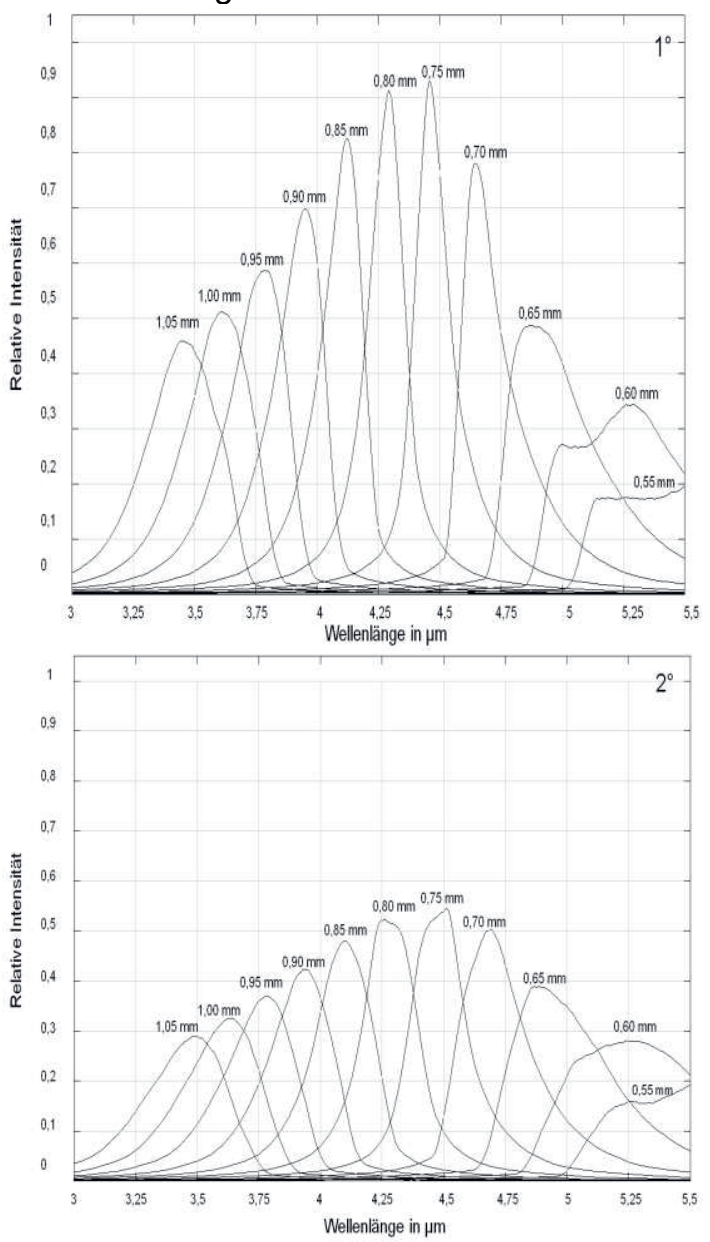

Abb. 4: Simulierte relative Intensität in Abhängigkeit von der Wellenlänge für ein System aus DOE1 und einer Blende von $30 \mu m$ Durchmesser für verschiedene Abstände mit Lichteinfall unter objektseitigen Winkeln von $1^{\circ}$ und $2^{\circ}$.

Die zuvor simulierten DOEs wurden mit Hilfe von Photolithographie und Inductively Coupled Plasmaätzprozess in einem zweistufigen Verfahren (siehe Abb. 5) erzeugt. Dazu wurde Photolack aufgebracht, dieser mit Hilfe einer ersten Maske strukturiert und die entsprechenden Strukturen durch einen ersten Ätzschritt ins Silizium übertragen. Daran schloss sich eine zweite Beschichtung mit Photolack, Belichtung mit einer zweiten Maske und ein zweiter Ätzschritt mit etwa der doppelten Tiefe statt. Die durch diesen Ätzprozess gefertigten DOEs wurden mittels Rasterelektronenmikroskop

Weißlichtinterferometer hinsichtlich ihrer Struktur untersucht (siehe Abb. 6).
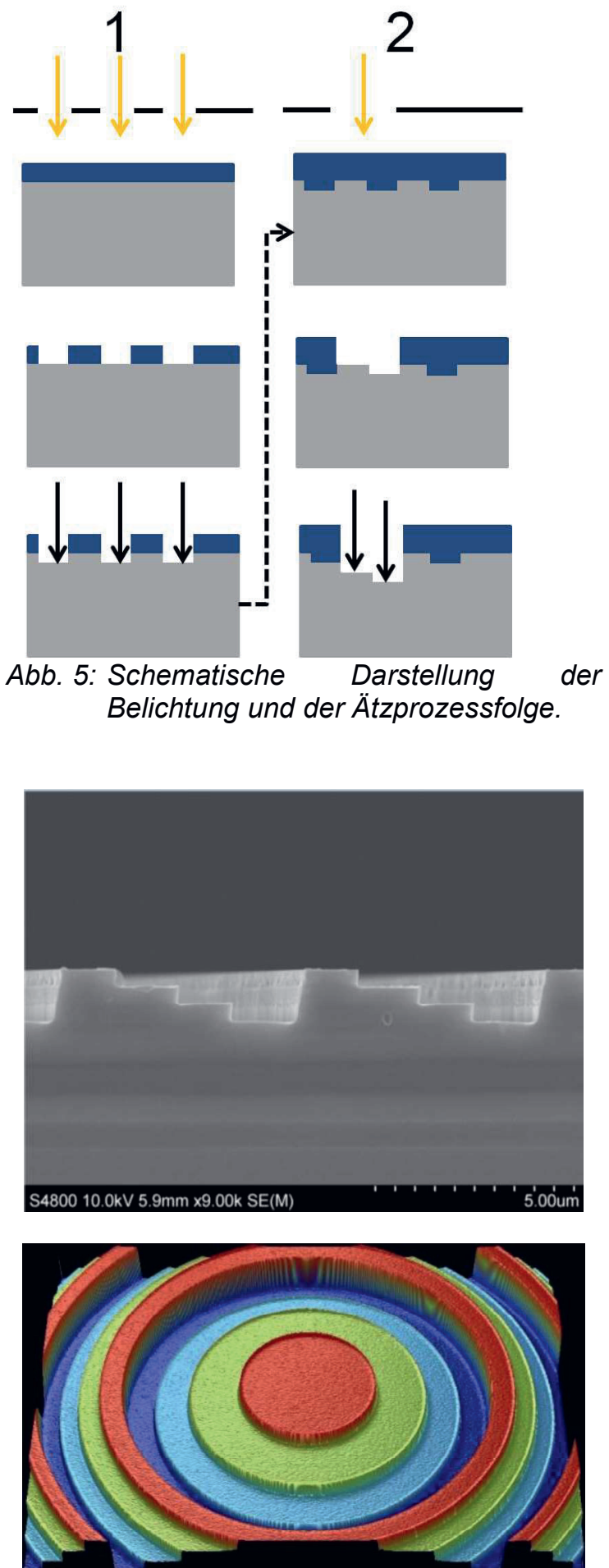

Abb. 6: Oben: Rasterelektronenmikrokopaufnahme eines vierstufigen DOE, Unten: Weißlichtinterferometeraufnahme eines vierstufigen DOE. 


\section{Design und Fertigung der Aktormembranen}

Ähnlich aufgebaute Gesamtsysteme mit aktorischen Membrandesigns wurden in [2] für die Erzeugung eines Mikrolinsenscanners verwendet. Die hier verwendeten Aktormembranfedern wurden im Design an die in [3] simulierten Federstrukturen angelehnt.

Abb. 7 zeigt Siliziummembranen mit Federstrukturen, welche im System als Membranen für den Aktor dienen.

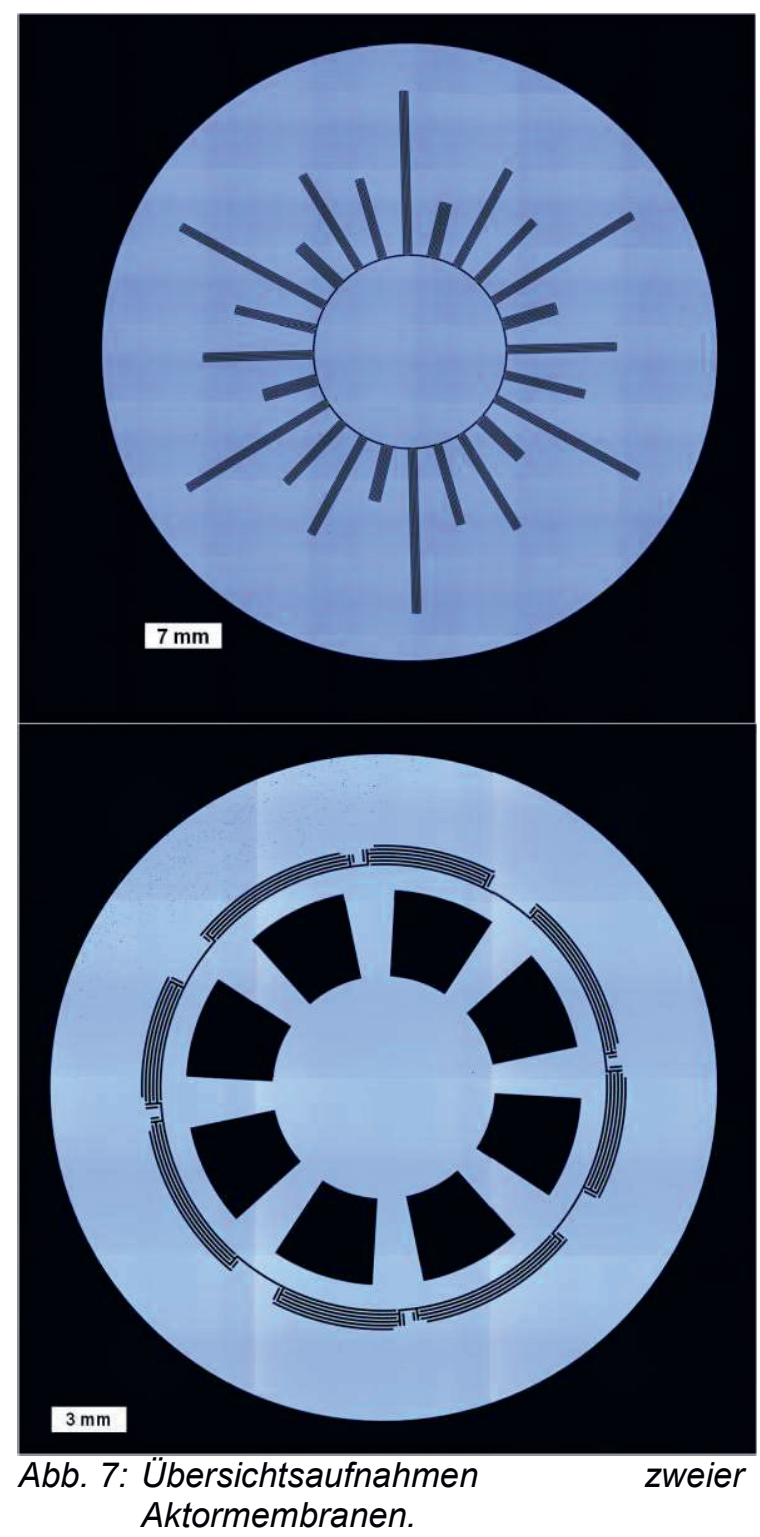

Detaillierte Aufnahmen der Federstrukturen der beiden Aktormembranen sind in Abb. 8 dargestellt.

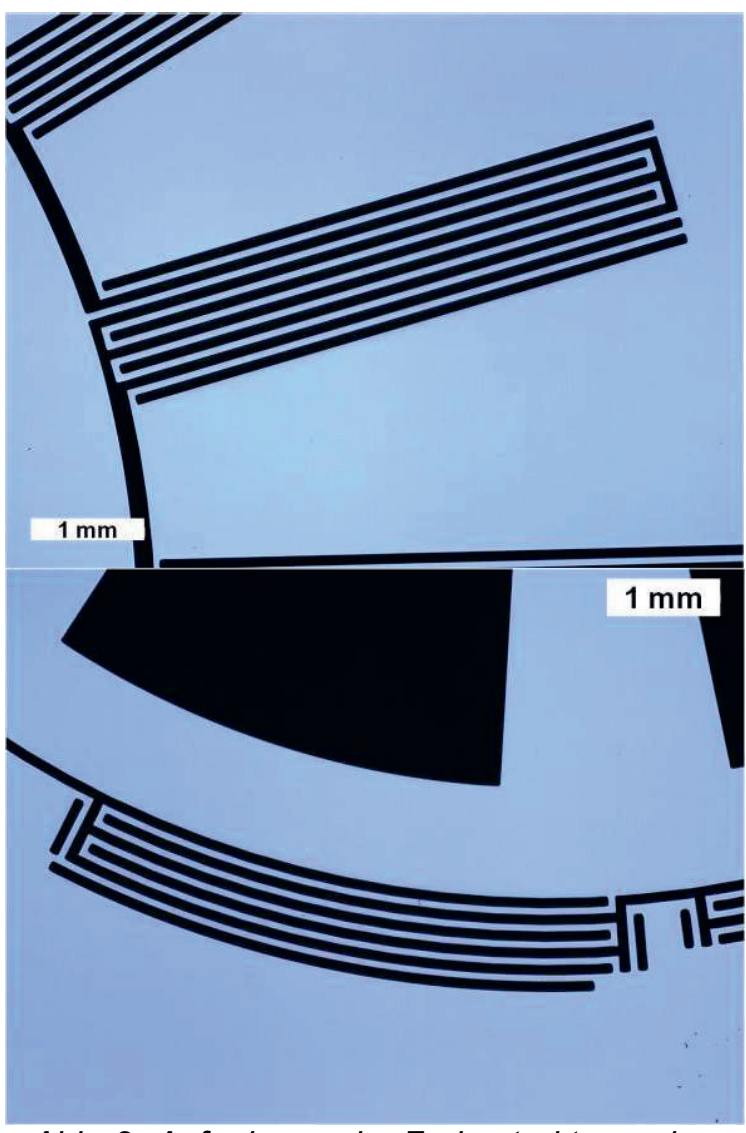

Abb. 8: Aufnahmen der Federstrukturen der Aktormembranen.

Auf Basis von mechanischen Simulationen konnten Abschätzungen zu erreichbaren Auslenkungen verschiedener Aktormembranen durchgeführt werden (siehe Abb. 9).

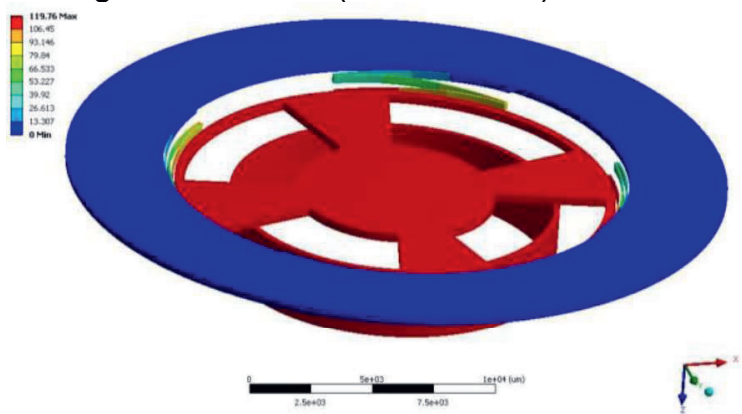

Abb. 9: Mechanische Simulation der Auslenkung einer Aktormembran mit Federstruktur.

Die Fertigung der Aktormembranen erfolgte mittels Inductively Coupled Plasmaätzprozess.

Rasterelektronenmikroskopaufnahmen der Federstrukturen sind in Abb.10 gezeigt. 


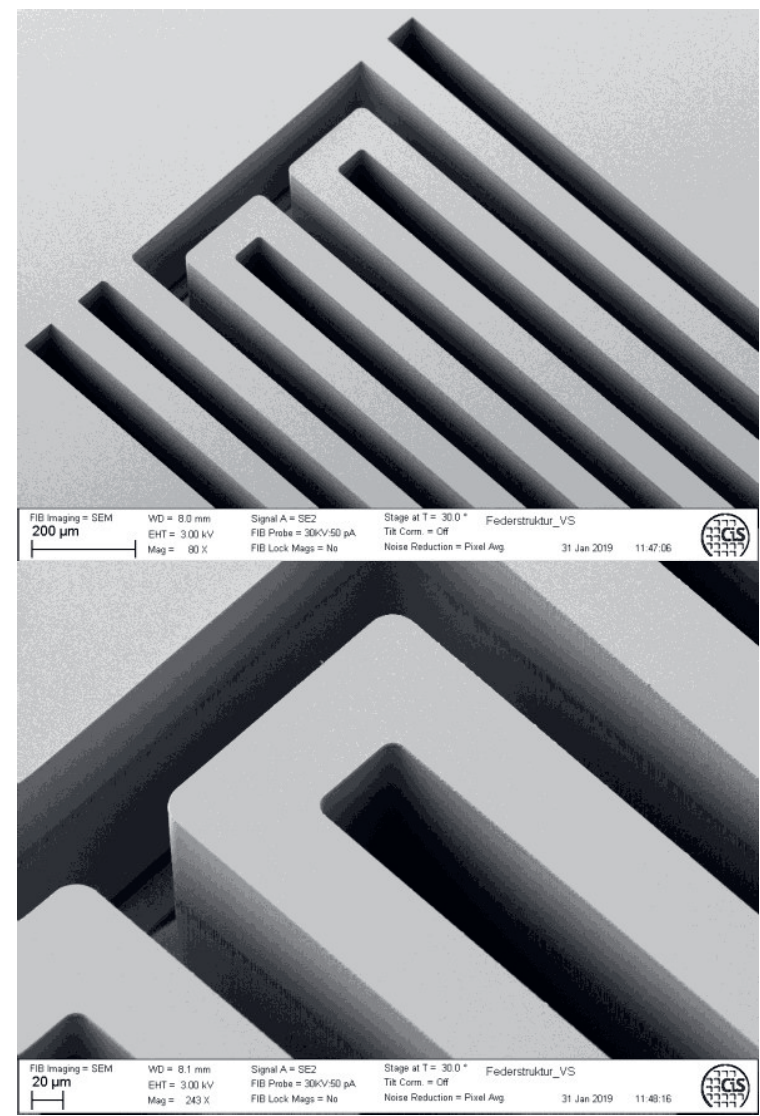

Abb.10: Rasterelektronenmikroskopaufnahmen von Federstrukturen.

\section{Danksagung}

Die Forschungs- und Entwicklungsarbeiten für das Aktorisches Miniaturspektrometer für die Gassensorik wurden im Projekt „AMIGA“ gefördert durch das Bundesministerium für Wirtschaft und Energie, InnoKom Förderkennzeichen MF170005

\section{Literaturnachweis}

[1] M. Hillenbrand, B. Mitschunas, C. Wenzel, A. Grewe , X. Ma,

P. Feßer, M. Bichra, and

S. Sinzinger, Adv. Opt. Techn. 1, 187194 (2012);

doi: 10.1515/aot-2012-0017

[2] S. Bargiel, C. Gorecki, T. Verdot, K. Laszczyk, J. Albero, and L. El Fissi, Procedia Engineering 5, 762-765 (2010); doi:10.1016/j.proeng.2010.09.220

[3] G. Barillaro, A. Molfese, A. Nannini, and F. Pieri, Journal of

Micromechanics and Microengineering 15, 736-746 (2005);

doi: 10.1088/0960-1317/15/4/010 